Research Paper

\title{
Down-expression of CD36 in pancreatic adenocarci- noma and its correlation with clinicopathological features and prognosis
}

\author{
Shengnan Jia\#, Liangjing Zhou\#, Tao Shen, Senhao Zhou, Guoping Ding ${ }^{凶}$, Liping Cao ${ }^{凶}$ \\ Department of General Surgery, Sir Run Run Shaw Hospital, School of Medicine, Zhejiang University, No. 3, Qingchun Road, Hangzhou, China \\ \# These authors have contributed equally to this work \\ $\square$ Corresponding author: Liping Cao, Guoping Ding, Department of General Surgery, Sir Run Run Shaw Hospital, School of Medicine, Zhejiang University, \\ Hangzhou, China. Phone (Fax): (+86) 0571-86090073; E-mail: caolipingzju@126.com; 11018045@zju.edu.cn \\ (C) Ivyspring International Publisher. This is an open access article distributed under the terms of the Creative Commons Attribution (CC BY-NC) license \\ (https://creativecommons.org/licenses/by-nc/4.0/). See http://ivyspring.com/terms for full terms and conditions.
}

Received: 2017.05.16; Accepted: 2017.08.31; Published: 2018.01.01

\begin{abstract}
Recent studies show that CD36 plays a key role in the occurrence and development of tumors, especially in the metastasis of tumors. However, the expression and role of CD36 has not been reported in pancreatic cancer. This study is aimed to explore the expression of CD36 in pancreatic cancer and corresponding non-tumor normal tissues, and its correlation with clinicopathological features and prognosis of pancreatic cancer patients. By analyzing the chip results of database GSE16515, we found that there was significant differential expression of CD36 in pancreatic cancer and corresponding non-tumor normal tissues. In this study, western blot and immunohistochemistry were used to show that the expression of CD36 in pancreatic cancer cells and tissues is significantly lower than that in corresponding non-tumor normal tissues. By statistically analyzing clinical and pathological data, we found that low expression of CD36 predicts lower TNM staging and CA19-9 levels, but larger tumor size and poor survival prognosis. These findings indicated that CD36 can be used as a predictor of clinicopathological features and prognosis, but the contradiction is worthy of our further study.
\end{abstract}

Key words: CD36, pancreatic cancer, metastasis, prognosis

\section{Introduction}

Pancreatic cancer is a common highly malignant tumor with a 5 -year survival rate of only $3 \%$ to $6 \%$, ranking as the $4^{\text {th }}$ most common cause of cancer-related death in the United States. Up to 85.3\% of deaths occur among newly diagnosed cases, ranking the first among all common malignant tumors [1]. In China, the incidence of pancreatic cancer is increasing by $2.3 \%$ each year, and the patients show a younger-age trend [2]. The prognosis of pancreatic cancer is very poor and more than $80 \%$ patients are in an advanced stage when they are diagnosed [3]. The opportunity for possibly curative radical surgery is lost owing to vascular invasion or distant metastasis. Further exploration of the mechanism of pancreatic cancer has important clinical significance to improve patient survival and quality of life.

CD36 is a member of the $\mathrm{B}$ class scavenger receptor family. It is a transmembrane glycoprotein receptor and is expressed on the surface of cells such as platelets [4], capillary endothelial cells [5], and macrophages [6]. As a cell surface receptor, CD36 can combine with ligands such as lipoproteins, serine phospholipids, and thrombospondin-1 (TSP1) to perform different functions $[7,8]$. CD36 is involved in the processes of lipid metabolism, long chain fatty acid adsorption, cell apoptosis clearing, and macrophage phagocytosis [9, 10]. A concentrated study of its expression regulation in the past suggested it may be a key factor in the formation of macrophage foam and atherosclerosis [11, 12]. However, recent studies found that CD36 also plays a 
key role in the occurrence and development of tumors, especially in the invasive capacity of metastatic tumors.

The studies conducted by the Spanish scientist Gloria Pascual et al. found that CD36 is a common marker of metastatic cells and is currently the first marker of metastatic specificity. Metastases of melanoma, breast cancer, ovarian cancer, bladder cancer, and lung cancer cells are dependent on CD36 [13]. In addition, studies report that CD36 plays an important role in tumor angiogenesis, epithelial stromal transformation, and other processes. The role of CD36 in tumors has attracted researchers' attention. However, the expression and role of CD36 has not been reported in pancreatic cancer. This study further explored the expression of CD36 in pancreatic cancer and corresponding non-tumor normal tissues and its correlation with clinicopathological features and the prognosis of pancreatic cancer, with the goal of understanding its role in the development and progression of pancreatic cancer.

\section{Materials and Methods}

\section{Cell culture}

Human pancreatic cancer cell line, including Panc-1, AsPC-1, SW1990, BxPC-3, MIAPaCa-2, and normal pancreatic ductal epithelial cells HPDE6-C7 were all obtained from Chinese Academy of Sciences (Shanghai, China). BxPC-3 and AsPC-1 cell lines were cultured in RPMI 1640 medium (Hyclone) containing $10 \%$ fetal bovine serum (FBS, Gibco, New York, USA). Panc-1, SW1990, MIAPaCa-2 and HPDE6-C7 cell lines were cultured in DMEM medium with 10\% FBS.

\section{Human tissue specimens and immunohistochemical analysis}

140 formalin-fixed and paraffin-embedded pancreatic ductal adenocarcinoma and adjacent normal pancreatic tissue specimens were obtained from pancreatic cancer patients between 2012 and 2015. The research protocol was reviewed and approved by the Research Ethics Committee of Sir Run Run Shaw Hospital, School of Medicine, Zhejiang University. All participants or their guardians gave written consent of their tissue samples and medical information to be used for scientific research. All cases were confirmed by pathological diagnosis and were analyzed according to 8th edition of UICC 2017 TNM classification. Immunohistochemical analyses of these specimens were conducted with anti-CD36 (ab133625; Abcam, 1:100) antibodies as described previously.

\section{Western blot analysis}

Cells were lysed with RIPA buffer (Sigma, St.Louis, MO, USA) containing a mixture of protease inhibitor cocktail kit (Thermo, Rockford, IL, USA). Then the lysates were cleared by centrifugation and the concentrations of proteins were measured by BCA protein assay kit (Pierce, USA). The proteins were denatured in $2 \times \mathrm{SDS}$ buffer at $95^{\circ} \mathrm{C}$, separated in $10 \%$ SDS-polyacrylamide gel electrophoresis (SDS-PAGE) and transferred onto polyvinylidene difluoride (PVDF) membrane (Millipore, Bedford, MA, USA). After being blocked with $5 \%$ skim milk powder for $1 \mathrm{~h}$ at room temperature, the membranes were probed with the CD36 (ab133625; Abcam;1:1000) antibodies. The samples were incubated with the secondary goat anti-rabbit antibodies conjugated with horseradish peroxidase (1:5 000, Pierce, USA) for $1 \mathrm{~h}$ at room temperature. The blots were visualized by enhanced chemiluminescence using Kodak X-OMAT LS film (Eastman Kodak, Rochester, NY).

\section{Statistical Analysis}

The data were compiled with the software package SPSS, version 19.0. Data were presented as mean \pm standard error (SE). Fisher's exact and $\chi^{2}$ tests were used to assess the associations between CD36 expressions and clinicopathological parameters. Univariate survival analysis was performed according to Kaplan-Meier, and differences in survival curves were assessed with the log-rank test. $P$ value $<0.05$ was considered as statistically significance.

\section{Results}

\section{Expression of CD36 in pancreatic cancer cells and tissues}

By analyzing chip results of 52 samples in the database GSE16515, including 36 cases of pancreatic cancer and 16 cases of corresponding non-tumor normal tissues, we found that CD36 has significant differential expression between the two and the expression of CD36 in pancreatic cancer is significantly lower than that in corresponding non-tumor normal tissues (Figure. 1A). Western blot method was used to detect the expression of CD36 in 5 pancreatic cancer cell lines and normal pancreatic ductal epithelial cells HPDE6-C7 as controls, we found that the expression of CD36 in pancreatic cancer cell lines is significantly decreased (Figure. 1B). In addition, we carried out CD36 immunohistochemical analysis of 140 cases of pancreatic cancer and normal pancreatic tissue specimens collected in our hospital and found that CD36 positive staining is mainly located on the cell membrane and in the cytoplasm. Among these samples, the positive expression rate of CD36 in pancreatic cancer tissues was $54.3 \%(76 / 140)$, which is lower than that in normal tissues $(89.3 \%, 125 / 140)$, and the difference was statistically significant (Figure. 1C/D, P < 0.05). 
$\mathbf{A}$

A GSE16515

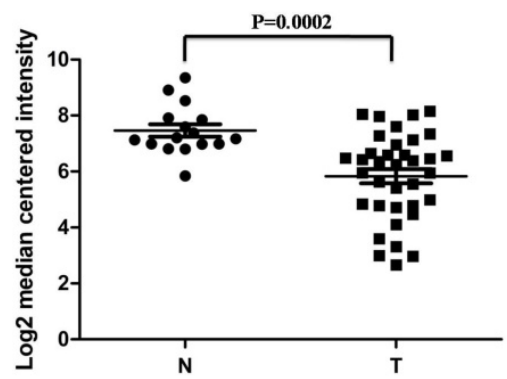

B

CD36

Tubulin
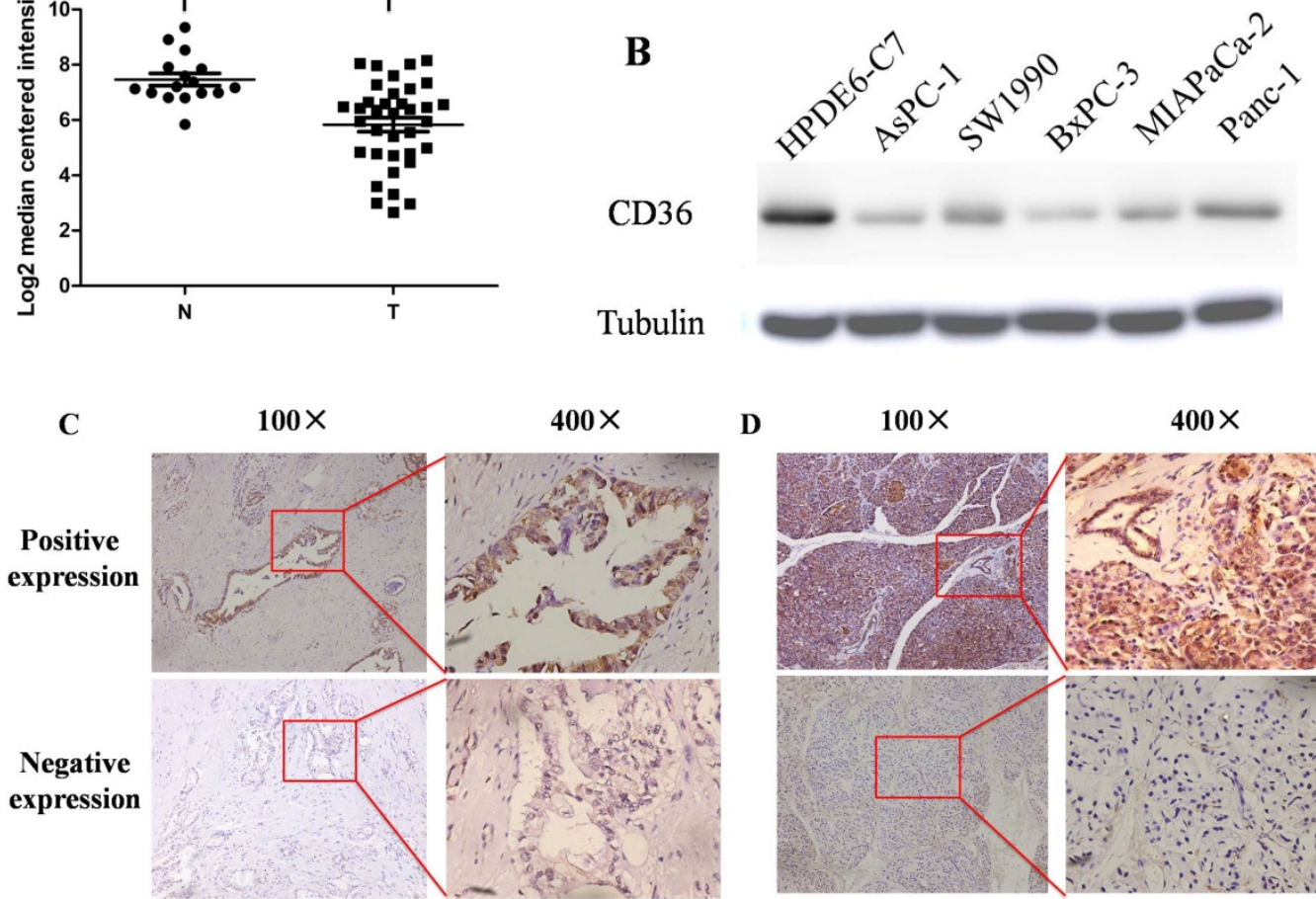

D

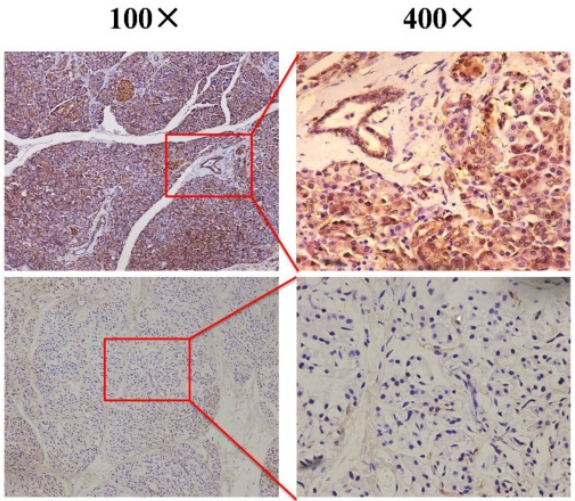

Figure. 1: Expression of CD36 in pancreatic cancer and normal pancreas. A: The mRNA expression of CD36 is down-regulated in pancreatic cancer tissues (T) compared with the adjacent non-tumor tissues (N) using the GSE16515 dataset. B: Western blot of CD36 in pancreatic cancer and normal pancreatic ductal epithelial cell lines. C,D: Immunohistochemistry was used to detect the expression of CD36 in pancreatic cancer tissues (C) and normal pancreatic tissues (D); the CD36 positive staining is mainly located on the cell membrane and in the cytoplasm.

\section{Correlation between CD36 expression and clinicopathological features}

We divided 140 patients into positive and negative groups according to the expression level of CD36. By statistically analyzing clinical and pathological data, we found that there is a correlation between CD36 expression level and tumor size, TNM stage and $\mathrm{T}$ stage. The TNM stage and $\mathrm{T}$ stage in the CD36 negative expression group was lower than that in the CD36 positive expression group. However, the tumor size of the CD36 negative expression group was significantly larger than that of the positive expression group and the difference was statistically significant (Figure. 2, Table 1, P < 0.05). In addition, the expression level of CD36 was positively correlated with the high density lipoprotein and CA19-9 expression level (Table 2, P $<0.05$ ). Similarly, we analyzed the correlation between CD36 expression levels and clinicopathologic parameters through the TCGA database. It was also found that patients in the CD36 negative expression group had lower TNM stage and larger size tumor (Table $3, \mathrm{P}<0.05$ ). In addition, we found that CD36 expression levels may also be related to $\mathrm{N}$ staging (Table 3, $\mathrm{P}<0.05$ ). These findings indicate that CD36 plays critical roles in pancreatic cancer development and progression and may be a valuable biomarker for this disease.

Table 1: Relationship between CD36 expression and clinicopathological features

\begin{tabular}{|c|c|c|c|c|}
\hline \multirow[t]{2}{*}{ Parameters } & \multirow{2}{*}{$\begin{array}{l}(\mathrm{n}) \\
140\end{array}$} & \multicolumn{2}{|c|}{ CD36 expressions } & \multirow[t]{2}{*}{$\mathrm{P}$ values } \\
\hline & & - & + & \\
\hline \multicolumn{5}{|l|}{ Age } \\
\hline$<60$ & 71 & 30 & 41 & 0.404 \\
\hline$\geq 60$ & 69 & 34 & 35 & \\
\hline \multicolumn{5}{|l|}{ Gender } \\
\hline Male & 83 & 34 & 49 & 0.173 \\
\hline Female & 57 & 30 & 27 & \\
\hline Poor & 71 & 32 & 39 & 0.877 \\
\hline Middle and high & 69 & 32 & 37 & \\
\hline \multicolumn{5}{|l|}{ Tumor size } \\
\hline$\leq 2 \mathrm{~cm}$ & 48 & 15 & 33 & $0.013^{*}$ \\
\hline$>2 \mathrm{~cm}$ & 92 & 49 & 43 & \\
\hline \multicolumn{5}{|l|}{$\mathrm{T}$ stage } \\
\hline $\mathrm{T} 1-2$ & 49 & 30 & 19 & $0.007^{*}$ \\
\hline T3-4 & 91 & 34 & 57 & \\
\hline \multicolumn{5}{|c|}{ Lymph node metastasis } \\
\hline No & 67 & 27 & 40 & 0.218 \\
\hline Yes & 73 & 37 & 36 & \\
\hline M0 & 126 & 57 & 69 & 0.734 \\
\hline M1 & 14 & 7 & 7 & \\
\hline \multicolumn{5}{|l|}{ TNM stage } \\
\hline I-II & 32 & 21 & 11 & $0.010^{*}$ \\
\hline III -IV & 108 & 43 & 65 & \\
\hline
\end{tabular}

${ }^{*} \mathrm{P}<0.05$ 
Table 2: Correlation between CD36 expression and clinical index

\begin{tabular}{llll}
\hline Parameters & \multicolumn{2}{l}{ CD36 expressions } & P values \\
\cline { 2 - 3 } & - & \multicolumn{2}{c}{+} \\
\hline Albumin, $g / \mathrm{L}$ & $37.92 \pm 4.24$ & $40.57 \pm 4.15$ & 0.071 \\
Total bilirubin, $\mu \mathrm{mol} / \mathrm{L}$ & $145.84 \pm 147.21$ & $104.89 \pm 128.09$ & 0.385 \\
Direct bilirubin, $\mu \mathrm{mol} / \mathrm{L}$ & $107.04 \pm 115.47$ & $70.78 \pm 87.89$ & 0.300 \\
Indirect bilirubin, $\mu$ mol/L & $38.80 \pm 38.04$ & $34.10 \pm 43.37$ & 0.738 \\
Triglyceride, $\mu$ mol/L & $2.09 \pm 1.23$ & $1.85 \pm 1.17$ & 0.571 \\
Total cholesterol, $\mu$ mol/L & $4.97 \pm 1.50$ & $5.14 \pm 1.22$ & 0.719 \\
High density lipoprotein, $\mu$ & $0.577 \pm 0.32$ & $0.92 \pm 0.47$ & $0.025^{*}$ \\
mol/L & & & \\
Low density lipoprotein, $\mu$ & $3.23 \pm 1.10$ & $3.06 \pm 0.80$ & 0.607 \\
mol/L & & & \\
Very low density lipoprotein, $\mu$ & $1.04 \pm 0.78$ & $1.01 \pm 0.67$ & 0.922 \\
mol/L & & & \\
Lipoprotein, mg/dl & $14.76 \pm 25.73$ & $22.35 \pm 24.52$ & 0.409 \\
CA19-9, IU/ml & $893.14 \pm 1584.86$ & $2861.27 \pm 4063$ & $0.037^{*}$ \\
CA-125, U/ml & $16.05 \pm 8.64$ & $30.67 \pm 29.33$ & 0.060 \\
AFP, ng/mL & $2.84 \pm 1.89$ & $3.09 \pm 1.95$ & 0.717 \\
CEA, ng/ml & $12.67 \pm 25.90$ & $41.78 \pm 147.44$ & 0.403 \\
\hline
\end{tabular}

* $\mathrm{P}<0.05$

Table 3: Relationship between CD36 expression and clinicopathological parameters in TCGA database

\begin{tabular}{|c|c|c|c|c|}
\hline \multirow[t]{2}{*}{ Parameters } & \multirow[t]{2}{*}{$\mathrm{n}$} & \multicolumn{2}{|c|}{ CD36 expressions } & \multirow[t]{2}{*}{$\mathrm{P}$ values } \\
\hline & & - & + & \\
\hline \multicolumn{5}{|l|}{ Age } \\
\hline$<60$ & 57 & 29 & 28 & 0.397 \\
\hline$\geq 60$ & 125 & 72 & 53 & \\
\hline \multicolumn{5}{|l|}{ Gender } \\
\hline Male & 100 & 61 & 39 & 0.099 \\
\hline Female & 82 & 40 & 42 & \\
\hline Poor & 52 & 28 & 24 & 0.843 \\
\hline Middle and high & 128 & 71 & 57 & \\
\hline \multicolumn{5}{|l|}{ Tumor size } \\
\hline$\leq 4 \mathrm{~cm}$ & 112 & 54 & 58 & $0.029^{*}$ \\
\hline$>4 \mathrm{~cm}$ & 56 & 37 & 19 & \\
\hline \multicolumn{5}{|l|}{ T stage } \\
\hline T1-2 & 31 & 14 & 17 & 0.201 \\
\hline T3-4 & 149 & 86 & 63 & \\
\hline \multicolumn{5}{|c|}{ Lymph node metastasis } \\
\hline No & 50 & 35 & 15 & $0.016^{*}$ \\
\hline Yes & 126 & 63 & 63 & \\
\hline M0 & 80 & 40 & 40 & 1.000 \\
\hline M1 & 5 & 3 & 2 & \\
\hline \multicolumn{5}{|l|}{ TNM stage } \\
\hline I-II A & 50 & 35 & 15 & $0.016^{*}$ \\
\hline II B-IV & 130 & 65 & 65 & \\
\hline
\end{tabular}

\section{CD36 expression and prognosis correlation}

We followed up the patients and found that the survival duration of the CD36 negative expression group was significantly lower than that of the CD36 positive expression group (Figure. $3 \mathrm{~A}, \mathrm{P}=0.024$ ). Meanwhile, we analyzed the correlation between survival prognosis and CD36 expression in pancreatic cancer patients in the TCGA database and found that the survival duration for the CD36 negative expression group was significantly shorter than that of the CD36 positive expression group (Figure. $3 \mathrm{~B}, \mathrm{P}=$ 0.010). This indicates that CD36 expression is an important factor affecting the prognosis of pancreatic cancer patients.

\section{Discussion}

In recent years, pancreatic cancer has become a malignant tumor with the fastest growing morbidity and mortality in the world, and a 5-year survival rate of only 3 to $6 \%$ [14]. An important feature of pancreatic cancer malignant biological behavior is its significant potential for invasion and metastasis. Extra-pancreatic spread can occur in the early stages of pancreatic cancer, which means more than $3 / 4$ of pancreatic cancer patients lose the opportunity of possibly curative radical surgery treatment [15]. Because pancreatic cancer has a hidden onset, rapid development of the disease, and a dangerous clinical course, most pancreatic cancer patients who are clinically treated present with an advanced stage and miss the best treatment opportunity. The metastasis mechanism, early diagnosis, prognostication, and anti-metastasis treatment of pancreatic cancer are problems that need to be solved in order to improve patient outcomes.

CD36 is a member of the B class scavenger receptor family. It is a transmembrane glycoprotein receptor and is expressed on surface of cells such as platelets, capillary endothelial cells, and macrophages. However, there are fewer studies on its expression in tumors; in particular, there is no report on its expression in pancreatic cancer. The results of this study show that CD36 has significantly lower expression in pancreatic cancer cells lines and tumor tissues. In addition, through the analysis of correlation between CD36 expression and clinicopathological features, we observed the TNM stage of the CD36 negative expression group was significantly declined than that of the CD36 positive expression group, but the tumor size in the CD36 negative expression group was significantly larger than that of the positive expression group. We considered that this discrepancy may be due to the decreased lymph node metastasis and infiltration of surrounding tissue in CD36 negative expression tissue that leading to the decline in $\mathrm{N}$ and $\mathrm{T}$ staging, thus resulting in a decline in TNM staging. Meanwhile, the expression of CA19-9 levels in CD36 negative expression tissue was also decreased, which means CD36 may be used as an important supplement for CA19-9 for predicting the outcomes of existing pancreatic cancer. Our study also found that there is a positive correlation between the expression of CD36 and the expression level of high-density lipoprotein, which is consistent with the results of a previous study by Jiang et al. that CD36 can promote cell uptake of high-density lipoprotein [16]. 
CD36 can combine with different ligands to perform different important functions. In recent years, the roles of CD36 in tumors have gradually been revealed, and especially in the metastasis of tumors, CD36 plays an important role. Nath et al. found that the CD36 signaling pathway promotes the uptake of fatty acids by hepatocellular carcinoma cells to drive epithelial mesenchymal transition in tumor cells, thus causing metastasis [17]. Thorne et al. stably transferred CD36 to MV3 melanoma cells without endogenous CD36 expression and found that expression of exogenous CD36 had no effect on the cell morphology and growth rate of MV3 melanoma cells, but their invasiveness increased [18]. According to the existing mechanism we may conclude that low expression of CD36 in pancreatic cancer is a protective factor. Interestingly, however, despite the CD36 negative expression indicates the lower TNM staging and lower CA19-9 expression levels, as well as previous studies have shown that over-expression of CD36 is associated with tumor progression, especially metastasis, this study first found that CD36 negative expression indicates a poor prognosis of survival, and confirmed by the TCGA database. This is in contradiction to what we have concluded earlier, and its further mechanism is still worth exploring.

In summary, our study found that the CD36 gene has a low expression level in pancreatic cancer. Low expression of CD36 predicts lower TNM staging and CA19-9 levels, but larger tumor size and poor survival prognosis. It can be used as a predictor of clinicopathological features and prognosis, but the contradiction is worthy of our further study.

\section{Acknowledgements}

This work was supported by the National Natural Science Foundation of China [grant number: 81572323]; A Project Supported by Scientific Research Fund of Zhejiang Provincial Education Department [grant number: Y201534615] and A Project Supported by Scientific Research Fund of Zhejiang Provincial Education Department [grant number: Y201534694].
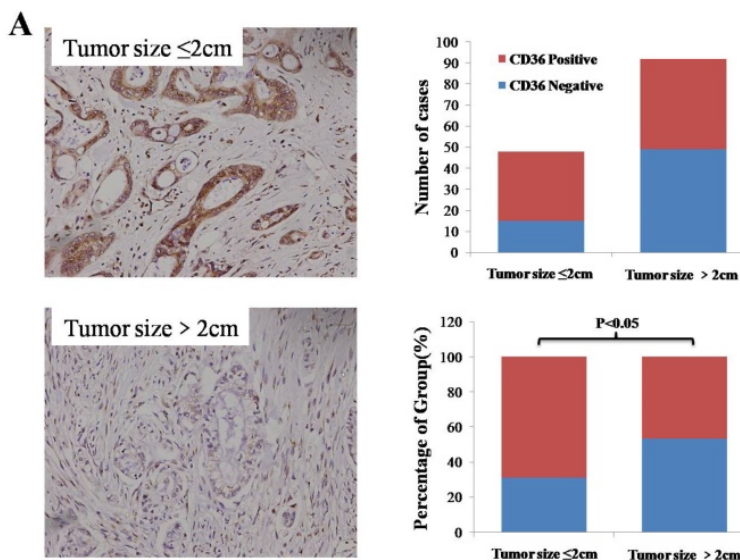

B

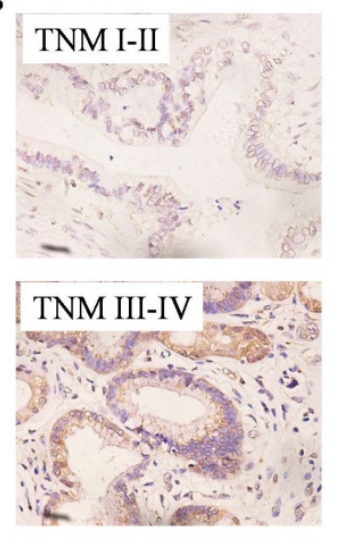

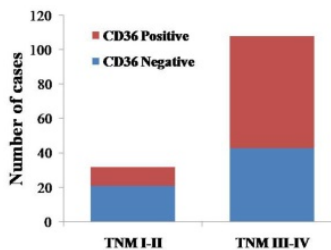

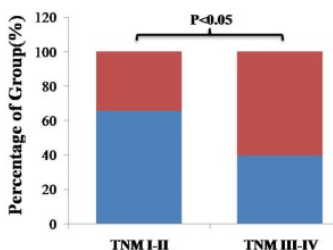

Figure. 2. Expression levels of CD36 in pancreatic cancer tissues with different tumor sizes and TNM stage. A: Negative association of CD36 expression with pancreatic tumor size. B: Positive association of CD36 expression with TNM stage in pancreatic cancer.

$\mathbf{A}$

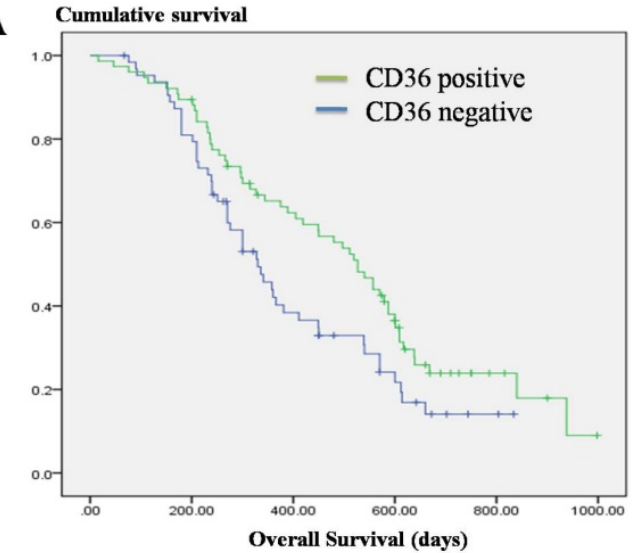

B

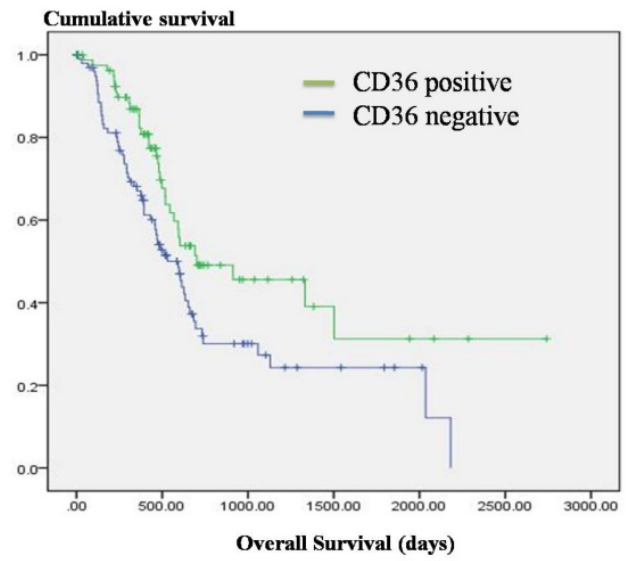

Figure. 3. Correlation of $C D 36$ expression and overall survival in pancreatic cancer. A: Overall survival in 140 pancreatic cancer patients. B: Overall survival in the TCGA datasets. 


\section{Competing Interests}

The authors have declared that no competing interest exists.

\section{References}

[1] Siegel RL, Miller KD, Jemal A. Cancer statistics, 2015. CA Cancer J Clin. 2015;65(1):5-29.

[2] Chen W, Zheng R, Baade PD, et al. Cancer statistics in China, 2015. CA Cancer J Clin. 2016;66(2):115-132

[3] Nagakawa T, Kobayashi H, Ueno K, et al. Clinical study of lymphatic flow to the paraaortic lymph nodes in carcinoma of the head of the pancreas. Cancer. 1994;73(4):1155-1162

[4] Yang M, Cooley BC, Li W, et al. Platelet CD36 promotes thrombosis by activating redox sensor ERK5 in hyperlipidemic conditions. Blood. 2017. pii: blood-2016-11-750133.

[5] Ramakrishnan DP, Hajj-Ali RA, Chen Y, et al. Extracellular Vesicles Activate a CD36-Dependent Signaling Pathway to Inhibit Microvascular Endothelial Cell Migration and Tube Formation. Arterioscler Thromb Vasc Biol. 2016;36(3):534-544.

[6] Yu M, Jiang M, Chen Y, et al. Inhibition of Macrophage CD36 Expression and Cellular Oxidized Low Density Lipoprotein (oxLDL) Accumulation by Tamoxifen: A PEROXISOME PROLIFERATOR-ACTIVATED RECEPTOR (PPAR) $\gamma$-DEPENDENT MECHANISM. J Biol Chem. 2016;291(33):16977-16989.

[7] Wong HS, Jaumouillé V, Freeman SA, et al. Chemokine Signaling Enhances CD36 Responsiveness toward Oxidized Low-Density Lipoproteins and Accelerates Foam Cell Formation. Cell Rep. 2016;14(12):2859-2871.

[8] Wang JM, Isenberg JS, Billiar TR, et al. Thrombospondin-1/CD36 pathway contributes to bone marrow-derived angiogenic cell dysfunction in type 1 diabetes via Sonic hedgehog pathway suppression. Am J Physiol Endocrinol Metab. 2013;305(12):E1464-1472.

[9] Glatz JF, Luiken JJ. From fat to FAT (CD36/SR-B2): Understanding the regulation of cellular fatty acid uptake. Biochimie. 2017; 136:21-26.

[10] Olonisakin TF, Li H, Xiong Z, et al. CD36 Provides Host Protection Against Klebsiella pneumoniae Intrapulmonary Infection by Enhancing Lipopolysaccharide Responsiveness and Macrophage Phagocytosis. J Infect Dis. 2016;214(12):1865-1875

[11] Sini S, Deepa D, Harikrishnan S, et al. High-density lipoprotein from subjects with coronary artery disease promotes macrophage foam cell formation: role of scavenger receptor CD36 and ERK/MAPK signaling. Mol Cell Biochem. 2017;427(1-2):23-34.

[12] Silverstein RL. Linking Metabolic Dysfunction to Atherosclerosis Via Activation of Macrophage CD36 Gene Transcription by Retinol Binding Protein-4. Circulation. 2017;135(14):1355-1356.

[13] Pascual G, Avgustinova A, Mejetta S, et al. Targeting metastasis-initiating cells through the fatty acid receptor CD36. Nature. 2017;541(7635):41-45.

[14] Kamisawa $\mathrm{T}$, Wood LD, Itoi $\mathrm{T}$, et al. Pancreatic cancer. Lancet. 2016;388(10039):73-85.

[15] Nakao A, Harada A, Nonami T, et al. Lymph node metastasis in carcinoma of the body and tail of the pancreas. Br J Surg. 1997;84(8):1090-1092.

[16] Jiang YG, Luo Y, He DL, et al. Role of Wnt/beta-catenin signaling pathway in epithelial-mesenchymal transition of human prostate cancer induced by hypoxia-inducible factor-1alpha. Int J Urol. 2007;14(11):1034-1039.

[17] Nath A, Li I, Roberts LR, et al. Elevated free fatty acid uptake via CD36 promotes epithelial-mesenchymal transition in hepatocellular carcinoma. Sci Rep. 2015; 5:14752.

[18] Thorne RF, Marshall JF, Shafren DR, et al. Theintegrins alpha3beta1 and alpha6beta1 physically and functionally associate with CD36 in human melanoma cells. Requirement for the extracellular domain OF CD36. J Biol Chem. 2000;275(45):35264-35275. 